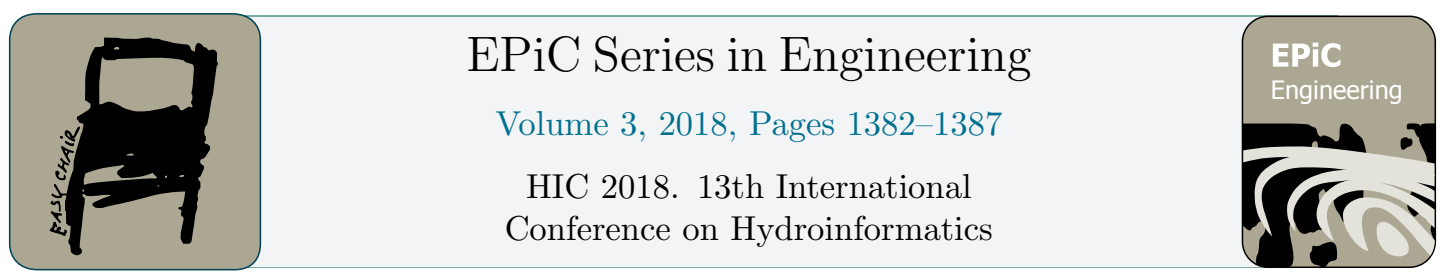

\title{
Pipe System Characteristics vs. Reliability of Transient Test-Based Techniques for Pipe Inspection
}

\author{
S. Meniconi ${ }^{1}$ and B. Brunone ${ }^{2}$ \\ 1 Department of Civil and Environmental Engineering, University of Perugia, 06125 Perugia, Italy \\ silvia.meniconi@unipg.it \\ 2 Department of Civil and Environmental Engineering, University of Perugia, 06125 Perugia, Italy \\ bruno.brunone@unipg.it
}

\begin{abstract}
In this paper the results of field and numerical experiments executed in a real transmission main are presented and discussed. Pressure waves injected into the pipe are generated by means of two quite different methodologies: pump shutdown and the Portable Pressure Wave Maker (PPWM) device.
\end{abstract}

\section{Introduction}

In drinkable water pipe systems, transmission mains (TMs) convey the whole discharge. As a consequence, even a small percentage of losses in TMs implies a large volume of non revenue water. However, until a few years ago, TMs were excluded from leak detection programs and in most cases the only action was to measure the inflow and outflow discharge. The two main reasons of such a behavior were: i) the unjustified conviction that leakage in TMs is negligible, and ii) the larger cost of inspection with respect to distribution networks. Nowadays things have changed radically: several technologies have been proposed for fault detection in TMs and experiments are in progress to test them. Roughly speaking, such technologies can be classified in two main families: the inline-type (ITT) and the transient test-based (TTBT) ones. To explore TMs, within the ITTs, sensors are inserted (e.g., puretechltd.com; echologics.com) whereas in TTBTs pressure waves are injected into the pipelines (e.g., [1]).

This paper clearly places in the research activity aiming the evaluation of the merits and drawbacks of TTBTs. Specifically, for given pipe system characteristics, the role played by the place where the pressure waves are injected with respect to the transient response of TMs is explored. The analysis is based on the field tests executed in a real TM with transients generated by means of two quite different methodologies: pump shutdown ([3]) and the Portable Pressure Wave Maker ([4]).

\section{Transient tests}

The examined TM is the iron pipe that supplies the city of Trento in the northeast of Italy (nominal diameter DN500, internal diameter, $D=506.6 \mathrm{~mm}$, wall thickness, $e=4.19 \mathrm{~mm}$, and

G. La Loggia, G. Freni, V. Puleo and M. De Marchis (eds.), HIC 2018 (EPiC Series in Engineering, vol. 3), pp. $1382-1387$ 


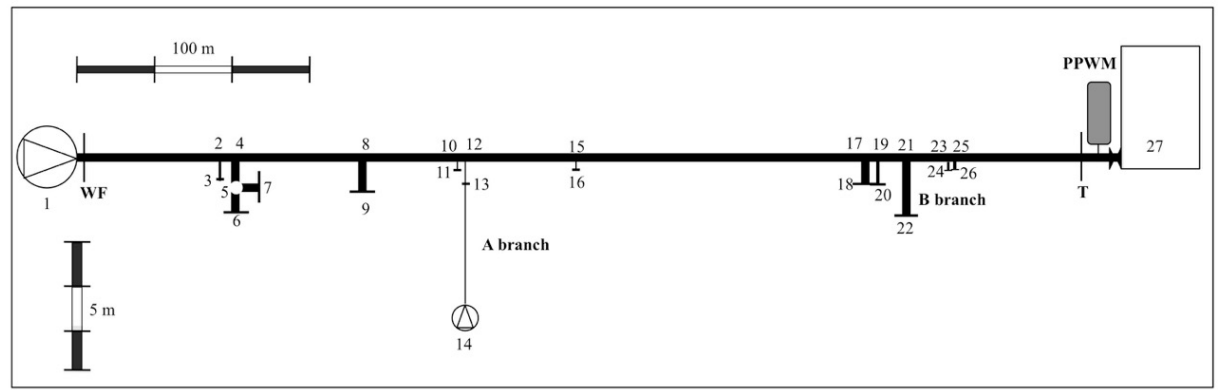

Figure 1: Trento TM layout (WF and T are the measurement sections at the well-field, and at 10000 reservoir, respectively; PPWM indicates the Portable Pressure Wave Maker device; note that a different length scale has been used for the main pipe and minor branches).

total length, $L=1321.97 \mathrm{~m}$ ). Such a pipe, managed by Novareti S.p.A., connects the Spini well-field (\#1 in Fig. 1) to 10000 reservoir (\#27 in Fig. 1). All the minor branches of the pipe (with a length, $L_{b}$, ranging between $0.7 \mathrm{~m}$ and $6.8 \mathrm{~m}$ ) are inactive; all branches are in steel with the exception of the 12-14 high density polyethylene (HDPE) pipe. Pressure signal, $H$, has been measured by means of piezoresistive transducers just downstream of the check valve at the well-field (section WF in Fig. 1) and the PPWM at 10000 reservoir (section T in Fig. 1), respectively. The first series of tests has been executed at the well-field, by stopping abruptly the electricity supply (Fig. 2), whereas the second series transients have been generated by means of the PPWM installed at a distance of $13.8 \mathrm{~m}$ from 10000 reservoir (Fig. 3). In both cases the repeatability of the tests has been checked by repeating the same maneuver three times (Figs. 2 and 3). The pressure wave speed, $a$, maybe the most crucial quantity within TTBTs, has been evaluated on the basis of the acquired pressure signals and the obtained value is compatible with the mechanical and geometrical characteristics of the pipe.

\section{Pressure signals analysis}

A main distinction of the analysis of the pressure signal concerns whether or not a numerical model simulating the transient test is used: in the first case an accurate model to simulate the observed transient data, known as the inverse transient analysis (ITA), is refined; in the second case a direct analysis of the pressure signal traces, referred to as the direct transient analysis (DTA), has been conducted [1]. The second approach - the most straightforward has been followed for analyzing the pressure signal acquired at section $\mathrm{T}$ (Fig. 2) concerning the transient in which a pressure wave is injected into the TM by means of the PPWM at 10000 reservoir (Fig. 4). As a first remark, the small amplitude (i.e., smaller than one meter of water column) of the pressure wave generated by such a device (Fig. 3) has to be pointed out. Secondly, because of the sharpness of the pressure wave (the duration of the maneuver is smaller than $0.1 \mathrm{~s}$ ), the detection of the minor branches closer to the injection point (i.e., 10000 reservoir) is quite clear. As an example, chains of maximum local moduli of the wavelet transform at $t=0.798 \mathrm{~s}$ and $t=1.755 \mathrm{~s}$ point out junction \#21 and \#12, respectively. The error in the localization of junction \#21 is equal to about $5.68 \%(296 \mathrm{~m}$, instead of $280 \mathrm{~m})$, whereas the error in the localization of junction \#12 is smaller (=1.63\%: $801 \mathrm{~m}$ instead of $814 \mathrm{~m}$ ). Moreover, the clear discontinuity at $t=1.85 \mathrm{~s}$, that corresponds to a singularity at a 


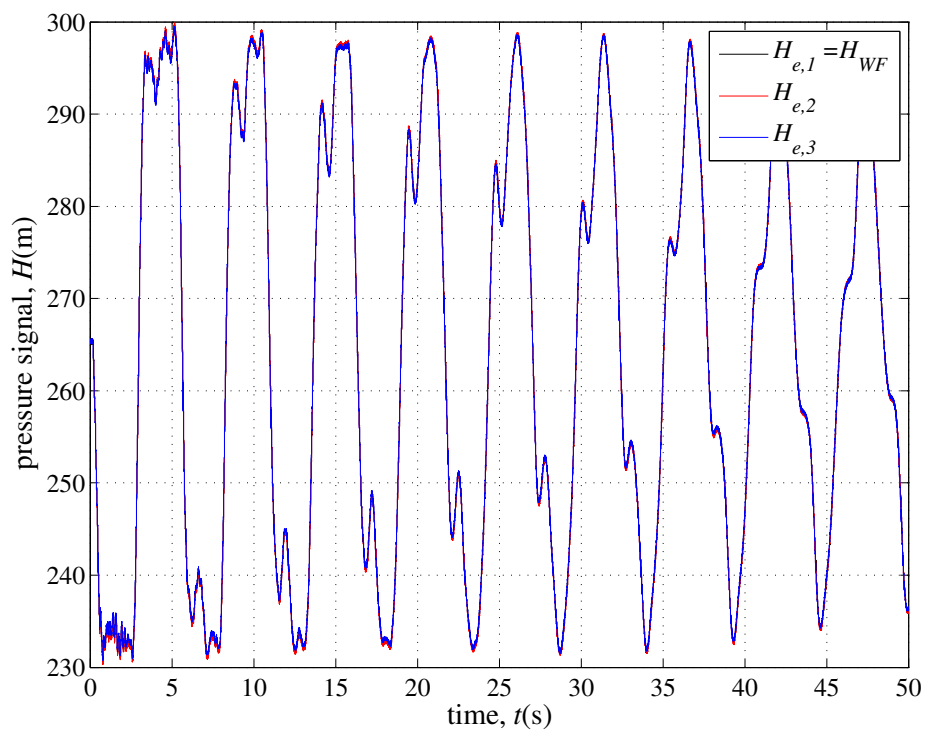

Figure 2: Pressure signals of the transient tests generated by pump shutdown at the Spini well-field (note the remarkable repeatability of the tests).

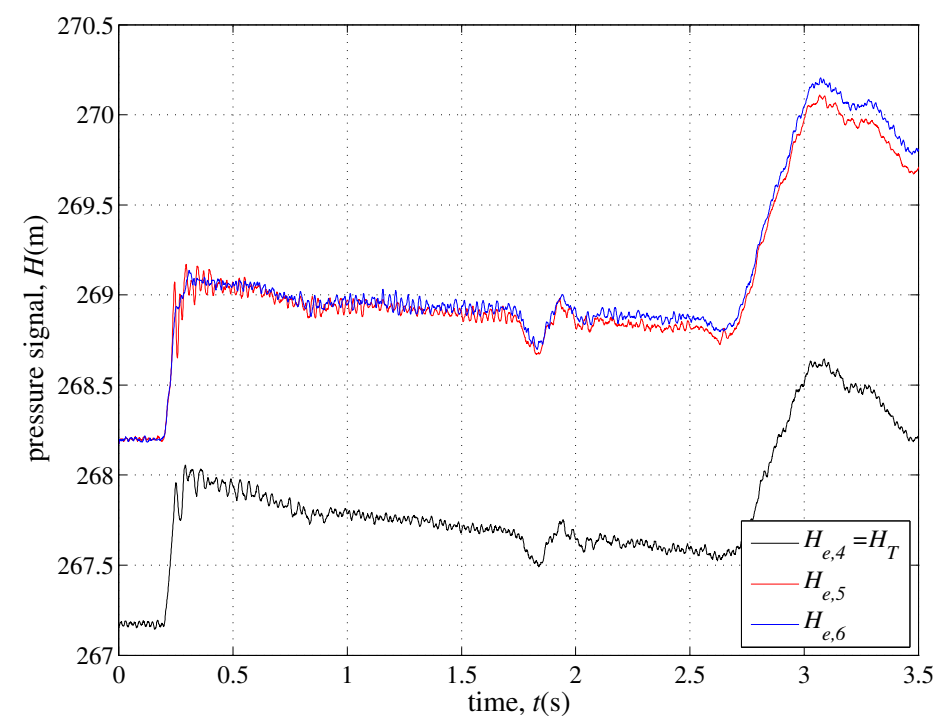

Figure 3: Pressure signals of the transient tests generated by means of the PPWM at 10000 reservoir (note the remarkable repeatability of the tests). 

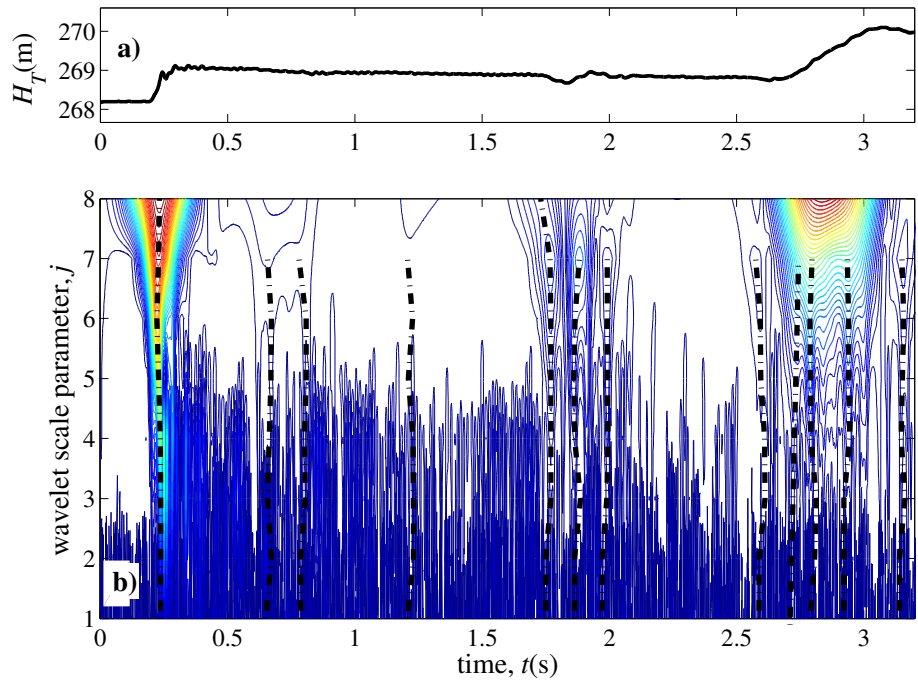

Figure 4: Wavelet analysis of the pressure signal of one of the the transient tests generated by means of the PPWM at 10000 reservoir.

distance of $19 \mathrm{~m}$ from junction \#12 (node \#14), has reveled the malfunctioning of the in-line valve \#13.

The analysis of the pressure signal, acquired during the transient test executed at the wellfield (Fig. 2) and measured at section WF, carried out by means of the wavelet analysis during the first characteristics time (Fig. 5), clearly points out the limits of such a kind of approach (DTA), because of the larger duration of the maneuver (about $0.5 \mathrm{~s}$ ), which affects the pressure signals and strongly influences the successive analysis. As a result, fault detection is not an easy task or better said, it is more difficult than expected and there is the need of using a numerical model to simulate the transients. As shown in [2], when complex pipe systems are examined, an integrated approach is needed by coupling DTA and ITA, where the characteristics of the defect (e.g., type, location, and severity) are the unknowns of the problem and are obtained within a calibration procedure by minimizing the difference between the measured data and the numerical model results.

As discussed in [3], the transient response of the Trento TM is largely different from the one expected if it behaved as a single pipe. An extensive analysis of the role of the minor branches, unsteady friction and viscoelasticity allowed to capture the main features of the examined pressure signals ([3]). The resulting numerical simulation (Fig. 6) is characterized by a quite large value $(=0.83)$ of the Nash-Sutcliffe efficiency coefficient. Moreover the malfunctioning of the in-line valve \#13 has been confirmed.

\section{Concluding Remarks}

Field and numerical experiments offered in this paper confirm potential of transient test-based techniques (TTBTs) which have arrows to their bow with respect to other methodologies (e.g., the the inline-type ones, ITT) for pressurized pipe inspection. In fact there is a link between 

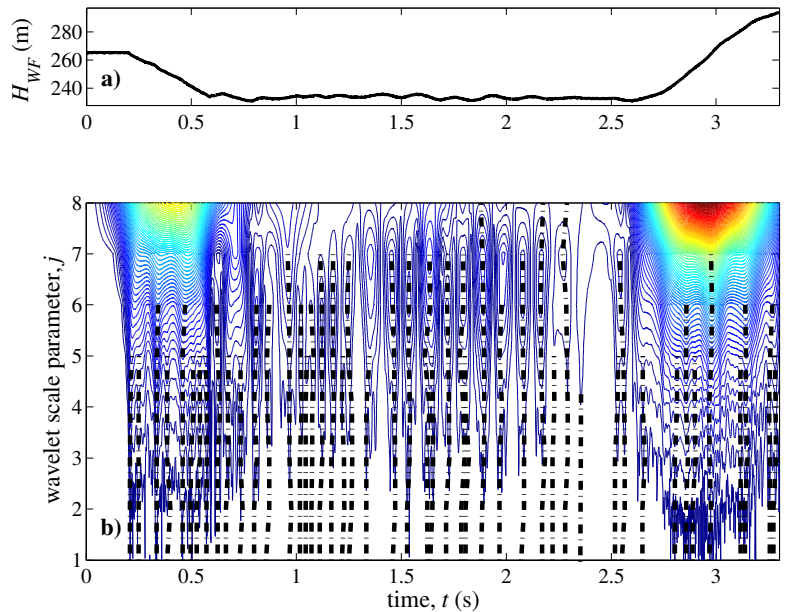

Figure 5: Wavelet analysis of the pressure signal of one of the transient tests generated by pump shutdown at the Spini well-field.

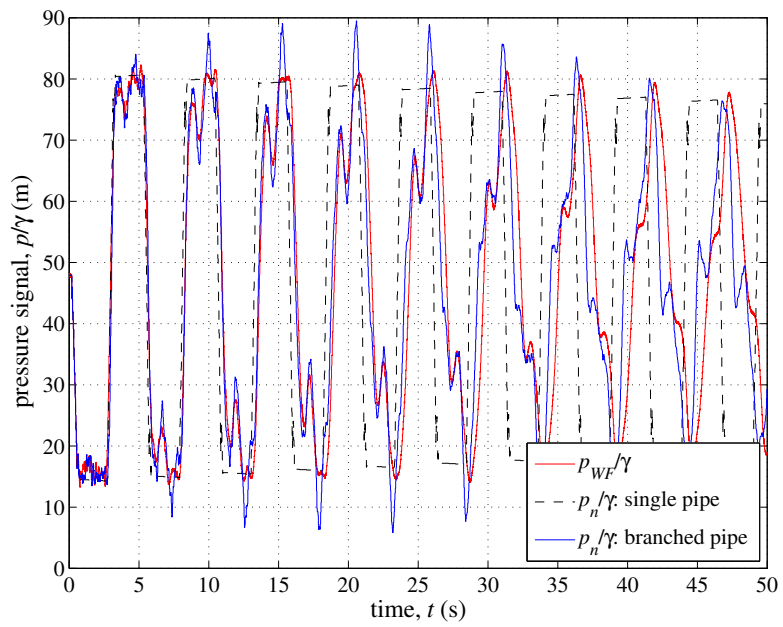

Figure 6: Transients generated by pump shut down at the Spini well field: experimental pressure signal, $p_{W F} / \gamma$, vs. numerical simulations, $p_{n} / \gamma$, of the single pipe and the branched pipe $(p=$ pressure, and $\gamma=$ specific weight). 
the minor branches - even if inactive - of the examined TM and the chains of the wavelet transform. In such a context, the role played by the characteristics of the maneuver generating the transient is also discussed. The importance of the sharpness of the injected pressure waves is once again pointed out as a crucial feature of TTBTs which can contribute significantly to the success of the inspection.

\section{Acknowledgments}

This research has been funded by the Hong Kong (HK) Research Grant Council Theme-Based Research Scheme and the HK University of Science and Technology (HKUST) under the project Smart Urban Water Supply System (Smart UWSS). Support from Italian MIUR and University of Perugia is acknowledged within the program Dipartimenti di Eccellenza 2018-2022.

\section{References}

[1] A.F. Colombo, P.J. Lee, and B.W. Karney. A selective literature review of transient-based leak detection methods. J Hydro-Environ Res, 2(4):212-227, 2009.

[2] S. Meniconi, B. Brunone, M. Ferrante, C. Capponi, C.A. Carrettini, C. Chiesa, D. Segalini, and E.A. Lanfranchi. Anomaly pre-localization in distribution-transmission mains. preliminary field tests in the milan pipe system. J. Hydroinform, 17:377-389, 2017.

[3] S. Meniconi, B. Brunone, and M. Frisinghelli. On the role of minor branches, energy dissipation, and small defects in the transient response of transmission mains. Water, 10(2):187, 2018.

[4] S. Meniconi, B. Brunone, M. Frisinghelli, E. Mazzetti, M. Larentis, and C. Costisella. Safe transients for pipe survey in a real transmission main by means of a portable device: the case study of the trento (i) supply system. Procedia Eng., 186:228-235, 2015. 